\title{
Approximation of Common Fixed Points of Pointwise Asymptotic Nonexpansive Maps in a Hadamard Space
}

\author{
Safeer Hussain Khan ${ }^{1}$, H. Fukhar-ud-din ${ }^{2}$ \\ ${ }^{1}$ Department of Mathematics, Statistics and Physics, Qatar University, Doha, Qatar \\ ${ }^{2}$ Department of Mathematics and Statistics, King Fahd University of Petroleum and Minerals, Dhahran, KSA \\ Email: safeer@qu.edu.qa, safeerhussain5@yahoo.com,hfdin@kfupm.edu.sa
}

Received July 18, 2012; revised September 18, 2012; accepted September 27, 2012

\begin{abstract}
We establish weak and strong convergence of Ishikawa type iterates of two pointwise asymptotic nonexpansive maps in a Hadamard space. For weak and strong convergence results, we drop "rate of convergence condition", namely

$\sum_{n=1}^{\infty}\left(c_{n}(x)-1\right)<\infty$, to answer in the affirmative to the open question posed by Tan and Xu [1] even in a general setup.
\end{abstract}

Keywords: Pointwise Asymptotic Nonexpansive Map; Common Fixed Point; Ishikawa Iteration Process; Strong Convergence; Weak Convergence

\section{Introduction}

A metric space $(X, d)$ is a length space if any two points of $X$ are joined by a rectifiable path (that is, a path of finite length) and the distance between any two points of $X$ is taken to be the infimum of the lengths of all rectifiable paths joining them. In this case, $d$ is known as length metric (otherwise an inner metric or intrinsic metric). In case, no rectifiable path joins two points of the space, the distance between them is taken to be $\infty$.

A geodesic path joining $x \in X$ to $y \in X$ (or, more briefly, a geodesic from $x$ to $y$ ) is a map $c$ from a closed interval $[0, l] \subset \mathbb{R}$ to $X$ such that $c(0)=x, \quad c(l)=y$, and $d\left(c(t), c\left(t^{\prime}\right)\right)=\left|t-t^{\prime}\right|$ for all $t, t^{\prime} \in[0, l]$. In particular, $c$ is an isometry and $d(x, y)=l$. The image $\alpha$ of $c$ is called a geodesic (or metric) segment joining $x$ and $y$. We say $X$ is: 1 ) a geodesic space if any two points of $X$ are joined by a geodesic and 2) uniquely geodesic if there is exactly one geodesic joining $x$ and $y$. for each $x, y \in X$, which we will denote by $[x, y]$, called the segment joining $x$ to $y$.

A geodesic triangle $\Delta\left(x_{1}, x_{2}, x_{3}\right)$ in a geodesic metric space $(X, d)$ consists of three points in $X$ (the vertices of $\Delta$ ) and a geodesic segment between each pair of vertices (the edges of $\Delta$ ). A comparison triangle for geodesic triangle $\Delta\left(x_{1}, x_{2}, x_{3}\right)$ in $(X, d)$ is a triangle $\bar{\Delta}\left(x_{1}, x_{2}, x_{3}\right):=\Delta\left(\bar{x}_{1}, \bar{x}_{2}, \bar{x}_{3}\right)$ in $\mathbb{R}^{2}$ such that $d_{\mathbb{R}^{2}}\left(\bar{x}_{i}, \bar{x}_{j}\right)=d\left(x_{i}, x_{j}\right)$ for $i, j \in\{1,2,3\}$ and such a triangle always exists (see [2]). A geodesic metric space is a $C A T(0)$ space if all geodesic triangles of appropriate size satisfy $C A T(0)$ comparison axiom: Let $\Delta$ be a geodesic triangle in $X$ and let $\bar{\Delta} \subset \mathbb{R}^{2}$ be a comparison triangle for $\Delta$. Then $\Delta$ is said to satisfy the CAT $(0)$ inequality if for all $x, y \in \Delta$ and all comparison points $\bar{x}, \bar{y} \in \bar{\Delta}$, we have

$$
d(x, y) \leq d(\bar{x}, \bar{y}) .
$$

For any $\alpha \in[0,1]$ and $x, y, z \in X$, Dhompongsa and Panyanak [3] modified the (CN) inequality of Bruhat and Tits $[4]$ as

$$
\begin{aligned}
& d^{2}(z, \alpha \oplus x(1-\alpha) y) \\
& \leq \alpha d^{2}(z, x)+(1-\alpha) d^{2}(z, y)-\alpha(1-\alpha) d^{2}(x, y) .
\end{aligned}
$$

If $\alpha=\frac{1}{2}$, then (1.1) reduces to the original $(\mathrm{CN})$ inequality of Bruhat and Tits [4].

Let us recall that a geodesic metric space is a CAT $(0)$ space if and only if it satisfies the $(C N)$ inequality (see [2, p. 163]). Complete $C A T(0)$ spaces are often called $\mathrm{Ha}$ damard spaces (see [5]). Moreover, if $X$ is a $C A T(0)$ metric space and $x, y, z \in X, \alpha \in[0,1]$ then there exists a unique point $\alpha x \oplus(1-\alpha) y \in[x, y]$ such that

$$
d(z, \alpha \oplus x(1-\alpha) y) \leq \alpha d(z, x)+(1-\alpha) d(z, y) .
$$

A subset $C$ of a $C A T(0)$ space $X$ is convex if for any $x, y \in C$, we have $[x, y] \subset C$.

In 2008, Kirk and $\mathrm{Xu}$ [6] studied (in Banach spaces) the existence of fixed points of asymptotic pointwise nonexpansive selfmap $T$ on $C$ defined by:

$d\left(T^{n}(x), T^{n}(y)\right) \leq c_{n}(x) d(x, y)$ for all $x, y \in C$ where $\limsup _{n \rightarrow \infty} c_{n}(x) \leq 1$. 
Their main result ([6], Theorem 3.5) states that every asymptotic pointwise nonexpansive selfmap of a nonempty closed bounded convex subset $C$ of a uniformly convex Banach space has a fixed point. This result of Kirk and $\mathrm{Xu}$ is a generalization of Goebel and Kirk fixed point theorem [7] for a narrower class of maps, the class of asymptotic nonexpansive maps, where (using our notation) every function $c_{n}$ is a constant function. In 2009, the results of [6] were extended to the case of metric spaces by Hussain and Khamsi [8]. As pointed out by Kirk and $\mathrm{Xu}$ in [6], asymptotic pointwise maps seem to be a natural generalization of nonexpansive maps. The conditions on $c_{n}$ can be, for instance, expressed in terms of the derivatives of iterations of $T$ for differentiable $T$.

$T$ is said to be asymptotic pointwise nonexpansive map if there exists a sequence of maps $a_{n}: C \rightarrow[0, \infty)$ such that $d\left(T^{n}(x), T^{n}(y)\right) \leq a_{n}(x) d(x, y)$ for all $x, y \in C$, $n \geq 1$, where $\limsup _{n \rightarrow \infty} a_{n}(x) \leq 1$. Denoting

$c_{n}(x)=\max \left(a_{n}(x), 1\right)$. Then note that without any loss of generality, $T$ is an asymptotic pointwise nonexpansive map if $d\left(T^{n}(x), T^{n}(y)\right) \leq c_{n}(x) d(x, y)$ for all $x, y \in C$, $n \geq 1$, where $c_{n}(x) \geq 1$ and $\lim _{n \rightarrow \infty} c_{n}(x)=1$. Moreover, we recall that $T: C \rightarrow C$ is uniformly $L$ Lipschitzian if for some $L>0$ we have that

$d\left(T^{n} x, T^{n} y\right) \leq L d(x, y)$, for $x, y \in K$ and $n \geq 1$; asymptotic nonexpansive if there is a sequence $\left\{k_{n}\right\} \subset$ $[1, \infty)$ with $\lim _{n \rightarrow \infty} k_{n}=1$ such that

$d\left(T^{n} x, T^{n} y\right) \leq k_{n} d(x, y)$, for all $x, y \in C$ and $n \geq 1$;

semi-compact (completely continuous) if for any bounded sequence $\left\{x_{n}\right\}$ in $C$ with $d\left(x_{n}, T x_{n}\right) \rightarrow 0$ as $n \rightarrow \infty$, there is a subsequence $\left\{x_{n_{i}}\right\}$ of $\left\{x_{n}\right\}$ such that $x_{n_{i}} \rightarrow$ $x^{*} \in C$ as $i \rightarrow \infty$.

Let $S, T: C \rightarrow C$ be asymptotic pointwise nonexpansive maps with function sequences $\left\{a_{n}(x) \geq 1\right\}$ and $\left\{b_{n}(x) \geq 1\right\}$ satisfying $\lim _{n \rightarrow \infty} a_{n}(x)=1$ and $\lim _{n \rightarrow \infty} b_{n}(x)=1$, respectively. Set

$$
\begin{aligned}
& c_{n}(x)=\max \left[a_{n}(x), b_{n}(x)\right] . \text { Then } \\
& \quad \lim _{n \rightarrow \infty} c_{n}(x)=\lim _{n \rightarrow \infty} a_{n}(x)=\lim _{n \rightarrow \infty} b_{n}(x)=1 .
\end{aligned}
$$

Therefore throughout the paper, we shall take $\tau(C)$ as the class of all pointwise asymptotic nonexpansive self maps $T$ on $C$ with function sequence $\left\{c_{n}(x) \geq 1\right\}$ with $\lim _{n \rightarrow \infty} c_{n}(x)=1$ for every $T \in \tau(C)$. Also $F$ will stand for the set of common fixed points of the two maps $S, T: C \rightarrow C$ We assume that $c_{n}$ is a bounded function for every $n \geq 1$ and all the functions $c_{n}$ are not bounded by a common constant, therefore a pointwise asymptotic nonexpansive map is not uniformly Lipschitzian. However, an asymptotic nonexpansive map is a pointwise asymptotic nonexpansive as well as uniformly Lipschitzian.

A strictly increasing sequence $\left\{n_{i}\right\}$ of natural num- bers is quasi-periodic if the sequence $\left\{n_{i+1}-n_{i}\right\}$ is bounded or equivalently if there exists a natural number $q$ such that any block of $q$ consecutive natural numbers must contain a term of the sequence $\left\{n_{i}\right\}$ The smallest of such numbers $q$ will be called a quasi-period of $\left\{n_{i}\right\}$.

Hussain and Khamsi [8] have shown that if $X$ is a Hadamard space and $C$ a nonempty bounded closed convex subset of $X$, then any pointwise asymptotic nonexpansive selfmap on $C$ has a fixed point. Moreover, this fixed point set is closed and convex. The proof of this important theorem is of the existential nature and does not describe any algorithm for constructing a fixed point of an asymptotic pointwise nonexpansive map. This paper aims at complementing their paper. It is also well known that the fixed point construction iteration processes for generalized nonexpansive maps have been successfully used to develop efficient and powerful numerical methods for solving various nonlinear equations.

Several authors have studied the generalizations of known iterative fixed point construction processes like the Mann process (see e.g. $[9,10]$ ) or the Ishikawa process (see e.g. [11]) to the case of asymptotic (but not pointwise asymptotic) nonexpansive maps. There is huge literature on the iterative construction of fixed points for asymptotic nonexpansive maps in Hilbert, Banach and metric spaces, see e.g. [1,3,7,9-25,27-32] and the references therein. Schu [32] proved the weak convergence of the Mann iteration process to a fixed point of asymptotic nonexpansive maps in uniformly convex Banach spaces with the Opial property [28] and the strong convergence for compact asymptotic nonexpansive maps in uniformly convex Banach spaces. Tan and $\mathrm{Xu}$ [1] proved the weak convergence of Mann and Ishikawa iteration processes for asymptotic nonexpansive maps in uniformly convex Banach spaces either satisfying the Opial condition or possessing Fréchet differentiable norm. Moreover, the rate of convergence condition namely $\sum_{n=1}^{\infty}\left(k_{n}-1\right)<\infty$ has remained in extensive use to prove both weak and strong convergence theorems to approximate fixed points of asymptotic nonexpansive maps in uniformly convex Banach spaces. Also Tan and Xu [1] remarked: we do not know whether our Theorem 3.1 remains valid if $k_{n}$ (the sequence associated with the asymptotic nonexpansive map $T$ ) is allowed to approach 1 slowly enough so that $\sum_{n=1}^{\infty}\left(k_{n}-1\right)$ diverges.

Recently Kozlowski [23] defined Mann type and Ishikawa type iterative processes to approximate fixed points of pointwise asymptotic nonexpansive maps in Banach spaces. We follow his idea and the concept of unique geodesic path denoted by $\alpha x \oplus(1-\alpha) y$ of two points $x, y$ in geodesic space and define Ishikawa type iterative process of two pointwise asymptotic nonexpansive maps in a geodesic space.

Let $C$ be a nonempty and convex subset of a geodesic 
space $X$ Let $S, T: C \rightarrow C$ be pointwise asymptotic nonexpansive maps and let $\left\{n_{k}\right\}$ be an increasing sequence of natural numbers and $0 \leq \alpha_{k}, \beta_{k} \leq 1$. Then the Ishikawa iteration process denoted by $I\left(S, T, \alpha_{k}, \beta_{k}, n_{k}\right)$ in a geodesic space $X$ is as under:

$$
\begin{aligned}
& x_{k+1}=\left(1-\alpha_{k}\right) x_{k} \oplus \alpha_{k} S^{n_{k}} y_{k} \\
& y_{k}=\left(1-\beta_{k}\right) x_{k} \oplus \beta_{k} T^{n_{k}} x_{k}(k \geq 1) .
\end{aligned}
$$

We say that $I\left(S, T, \alpha_{k}, \beta_{k}, n_{k}\right)$ is well-defined if $\limsup _{k \rightarrow \infty} c_{n_{k}}\left(x_{k}\right)=1$.

\section{Fixed Point Approximation}

Following the investigations of Hussain and Khamsi [8], the existence of the fixed point of pointwise asymptotic nonexpansive map can not be achieved without its bounded domain. We shall follow them for the purpose. We start with proving the following lemma.

Lemma 2.1. Let $C$ be a nonempty, bounded, closed and convex set in a geodesic space $X$ and let

$S, T \in \tau(C)$. Let $\left\{n_{k}\right\}$ be such that the sequence $\left\{x_{k}\right\}$ in (1.2) is well defined. If the set $\Omega=\left\{j: n_{j+1}=n_{j}+1\right\}$ is quasiperiodic and

$$
\lim _{k \rightarrow \infty} d\left(x_{k}, S^{n_{k}} x_{k}\right)=0=\lim _{k \rightarrow \infty} d\left(x_{k}, T^{n_{k}} x_{k}\right),
$$

then

$$
\lim _{k \rightarrow \infty} d\left(x_{k}, S^{n_{k}} x_{k}\right)=0=\lim _{k \rightarrow \infty} d\left(x_{k}, T^{n_{k}} x_{k}\right)=0 .
$$

Proof. Set $c_{k}=d\left(x_{k}, S^{n_{k}} x_{k}\right)$ and $d_{k}=d\left(x_{k}, S^{n_{k}} x_{k}\right)$. From

$$
\begin{aligned}
d\left(x_{k}, y_{k}\right) & =d\left(x_{k},\left(1-\beta_{k}\right) x_{k} \oplus \beta_{k} T^{n_{k}} x_{k}\right) \\
& \leq \beta_{k} d\left(x_{k}, T^{n_{k}} x_{k}\right) \rightarrow 0 \text { as } k \rightarrow \infty,
\end{aligned}
$$

we have

$$
\lim _{k \rightarrow \infty} d\left(x_{k}, y_{k}\right)=0 \text {. }
$$

Also

$$
\begin{aligned}
& d\left(x_{k}, y_{k+1}\right)=d\left(x_{k},\left(1-\alpha_{k}\right) x_{k} \oplus \alpha_{k} S^{n_{k}} x_{k}\right) \\
& \leq \alpha_{k} d\left(x_{k}, S^{n_{k}} x_{k}\right) \leq d\left(x_{k}, S^{n_{k}} x_{k}\right)+d\left(S^{n_{k}} x_{k}, S^{n_{k}} y_{k}\right) \\
& \leq d\left(x_{k}, S^{n_{k}} x_{k}\right)+c_{n_{k}}\left(x_{k}\right) d\left(x_{k}, y_{k}\right) .
\end{aligned}
$$

$$
\begin{aligned}
d^{2}\left(x_{k+1}, p\right)= & d^{2}\left(\left(1-\alpha_{k}\right) x_{k} \oplus \alpha_{k} S^{n_{k}} y_{k}, p\right) \leq \alpha_{k} d^{2}\left(S^{n_{k}} y_{k}, p\right)+\left(1-\alpha_{k}\right) d^{2}\left(x_{k}, p\right)-\alpha_{k}\left(1-\alpha_{k}\right) d^{2}\left(x_{k}, S^{n_{k}} y_{k}\right) \\
\leq & \alpha_{k} c_{n_{k}}^{2}(p) d^{2}\left(y_{k}, p\right)+\left(1-\alpha_{k}\right) d^{2}\left(x_{k}, p\right)-\alpha_{k}\left(1-\alpha_{k}\right) d^{2}\left(x_{k}, S^{n_{k}} y_{k}\right) \\
= & \alpha_{k} c_{n_{k}}^{2}(p) d^{2}\left(\left(1-\beta_{k}\right) x_{k} \oplus \beta_{k} T^{n_{k}} x_{k}, p\right)+\left(1-\alpha_{k}\right) d^{2}\left(x_{k}, p\right)-\alpha_{k}\left(1-\alpha_{k}\right) d^{2}\left(x_{k}, S^{n_{k}} x_{k}\right) \\
\leq & {\left[\alpha_{k} \beta_{k} c_{n_{k}}^{4}(p)+\alpha_{n} c_{n_{k}}^{2}(p)\left(1-\beta_{k}\right)+\left(1-\alpha_{k}\right)\right] d^{2}\left(x_{k}, p\right) } \\
& -\alpha_{k} c_{n_{k}}^{2}(p) \beta_{k}\left(1-\beta_{k}\right) d^{2}\left(x_{k}, T^{n_{k}} x_{k}\right)-\alpha_{k}\left(1-\alpha_{k}\right) d^{2}\left(x_{k}, S^{n_{k}} y_{k}\right) \\
\leq & {\left[\alpha_{k} \beta_{k} c_{n_{k}}^{4}(p)+\alpha_{n} c_{n_{k}}^{4}(p)\left(1-\beta_{k}\right)+\left(1-\alpha_{k}\right) c_{n_{k}}^{4}(p)\right] d^{2}\left(x_{k}, p\right) } \\
& -\alpha_{k} c_{n_{k}}^{2}(p) \beta_{k}\left(1-\beta_{k}\right) d^{2}\left(x_{k}, T^{n_{k}} x_{k}\right)-\alpha_{k}\left(1-\alpha_{k}\right) d^{2}\left(x_{k}, S^{n_{k}} y_{k}\right) .
\end{aligned}
$$

therefore taking limsup on both the sides of inequality (2.5) and using (2.1) and (2.4), we get $\limsup _{k \rightarrow \infty} d\left(x_{k}, S x_{k}\right) \leq 0$ and hence

$$
\lim _{k \rightarrow \infty} d\left(x_{k}, S x_{k}\right)=0 \text {. }
$$

$$
\lim _{k \rightarrow \infty} d\left(x_{k}, T x_{k}\right)=0
$$

That is,

$$
\lim _{k \rightarrow \infty} d\left(x_{k}, S x_{k}\right)=0=\lim _{k \rightarrow \infty} d\left(x_{k}, T x_{k}\right) .
$$

Remark 2.2. Lemma 2.1 extends the corresponding Lemma 3 of Khan and Takahashi [22] from Lipschitzian to non-Lipschitzian maps.

Lemma 2.3. Let $C$ be a nonempty, bounded, closed and convex subset of a Hadamard space $X$ and let $S, T \in \tau(C)$. Let $\alpha_{k}, \beta_{k} \in(\delta, 1-\delta)$ for some $\delta \in\left(0, \frac{1}{2}\right)$ and $\left\{n_{k}\right\}$ be such that the sequence $\left\{x_{k}\right\}$ in (1.3) is well-defined. If the set $\Omega=\left\{j: n_{j+1}=n_{j}+1\right\}$ is quasiperiodic and $F \neq \varnothing$, then

$$
\lim _{k \rightarrow \infty} d\left(x_{k}, S x_{k}\right)=0=\lim _{k \rightarrow \infty} d\left(x_{k}, T x_{k}\right) .
$$

Proof. Let $p \in F$. Then use (CN) inequality (1.1) for the scheme (1.2) to have 
Since $C$ is bounded, there exists

$B_{r}\left[x_{0}\right]=\left\{x \in X: d\left(x, x_{0}\right) \leq r\right\} \quad$ such that $C \subset B_{r}\left[x_{0}\right]$ for some $r>0$ Therefore the above inequality becomes

$$
\begin{aligned}
d^{2}\left(x_{k+1}, p\right) \leq & d^{2}\left(x_{k}, p\right)+r^{2}\left(c_{n_{k}}^{4}(p)-1\right) \\
& -\delta^{3} d^{2}\left(x_{k}, T^{n_{k}} x_{k}\right)-\delta^{2} d^{2}\left(x_{k}, S^{n_{k}} y_{k}\right) .
\end{aligned}
$$

From (2.6), the following two inequalities are obtained

$$
\begin{aligned}
d^{2}\left(x_{k+1}, p\right) \leq & d^{2}\left(x_{k}, p\right)+r^{2}\left(c_{n_{k}}^{4}(p)-1\right) \\
& -\delta^{2} d^{2}\left(x_{k}, S^{n_{k}} y_{k}\right),
\end{aligned}
$$

and

$$
\begin{aligned}
d^{2}\left(x_{k+1}, p\right) \leq & d^{2}\left(x_{k}, p\right)+r^{2}\left(c_{n_{k}}^{4}(p)-1\right) \\
& -\delta^{3} d^{2}\left(x_{k}, S^{n_{k}} y_{k}\right) .
\end{aligned}
$$

Now, we prove that

$$
\lim _{k \rightarrow \infty} d\left(x_{k}, S^{n_{k}} y_{k}\right)=0=\lim _{k \rightarrow \infty} d\left(x_{k}, T^{n_{k}} x_{k}\right) .
$$

First assume limsup ${ }_{k \rightarrow \infty} d\left(x_{k}, S^{n_{k}} y_{k}\right)>0$ Then there exists a subsequence(use the same notation for subsequence as for the sequence) of $\left\{x_{k}\right\}$ and $\mu>0$ such that $d\left(x_{k}, S^{n_{k}} y_{k}\right) \geq \mu>0$.

From (2.7), it follows that

$$
\begin{aligned}
& d^{2}\left(x_{k+1}, p\right) \leq d^{2}\left(x_{k}, p\right)+r^{2}\left(c_{n_{k}}^{4}(p)-1\right)-(\delta \mu)^{2} \\
& =d^{2}\left(x_{k}, p\right)+r^{2}\left(\left(c_{n_{k}}^{4}(p)-1\right)-\frac{(\delta \mu)^{2}}{2 r^{2}}\right) \\
& =d^{2}\left(x_{k}, p\right)+r^{2}\left(\left(c_{n_{k}}^{4}(p)-1\right)-\frac{(\delta \mu)^{2}}{2 r^{2}}\right)-\frac{(\delta \mu)^{2}}{2} .
\end{aligned}
$$

Since $c_{n_{k}}^{4}(p) \rightarrow 1$ and $\frac{(\delta \mu)^{2}}{2 r^{2}}>0$, so there exists $k_{0} \geq 1$ such that $\left(c_{n_{k}}^{4}(p)-1\right)<\frac{(\delta \mu)^{2}}{2 r^{2}}$ for all $k \geq k_{0}$.

Hence the above inequality reduces to

$$
\frac{(\delta \mu)^{2}}{2} \leq d^{2}\left(x_{k}, p\right)-d^{2}\left(x_{k+1}, p\right) .
$$

Let $l \geq k_{0}$ be any positive integer. Then from (2.10), we have

$$
\begin{aligned}
\frac{(\delta \mu)^{2}}{2}\left(l-k_{0}\right) & \leq d^{2}\left(x_{k_{0}}, p\right)-d^{2}\left(x_{l+1}, p\right) \\
& \leq d^{2}\left(x_{k_{0}}, p\right)<\infty .
\end{aligned}
$$

Letting $l \rightarrow \infty$ in (2.11), we get

$$
\infty \leq d^{2}\left(x_{k_{0}}, p\right)<\infty \text {, }
$$

a contradiction.
Hence

$$
\limsup _{k \rightarrow \infty} d\left(x_{k}, S^{n_{k}} y_{k}\right) \leq 0 .
$$

Consequently, we have

$$
\lim _{k \rightarrow \infty} d\left(x_{k}, S^{n_{k}} y_{k}\right)=0 .
$$

Following the similar procedure of proof with (2.8), we conclude

$$
\lim _{k \rightarrow \infty} d\left(x_{k}, T^{n_{k}} x_{k}\right)=0
$$

Since

$$
\begin{aligned}
d\left(x_{k}, S^{n_{k}} x_{k}\right) & \leq d\left(x_{k}, S^{n_{k}} y_{k}\right)+d\left(S^{n_{k}} x_{k}, S^{n_{k}} y_{k}\right) \\
& \leq d\left(x_{k}, S^{n_{k}} y_{k}\right)+b_{n_{k}}\left(x_{k}\right) d\left(x_{k}, y_{k}\right),
\end{aligned}
$$

therefore with the help of (2.2) and (2.12), we get

$$
\lim _{k \rightarrow \infty} d\left(x_{k}, S^{n_{k}} x_{k}\right)=0 .
$$

Finally, Lemma 2.1 appeals that

$$
\lim _{k \rightarrow \infty} d\left(x_{k}, S x_{k}\right)=0=\lim _{k \rightarrow \infty} d\left(x_{k}, T x_{k}\right) .
$$

Let $\left\{x_{k}\right\}$ be a bounded sequence in a metric space $X$. For $x \in X$, define $r\left(x,\left\{x_{k}\right\}\right)=\limsup _{k \rightarrow \infty} d\left(x_{k}, x\right)$. The asymptotic radius $r\left(\left\{x_{k}\right\}\right)$ of $\left\{x_{k}\right\}$ is given by:

$$
r\left(\left\{x_{k}\right\}\right)=\inf \left\{r\left(x,\left\{x_{k}\right\}\right): x \in X\right\} .
$$

A bounded sequence $\left\{x_{k}\right\}$ in $X$. is regular if $r\left(\left\{x_{k}\right\}\right)=r\left(\left\{u_{k}\right\}\right)$ for every subsequence $\left\{u_{k}\right\}$ of $\left\{x_{k}\right\}$

The asymptotic center of a bounded sequence $\left\{x_{k}\right\}$ with respect to $C \subseteq X$ is defined

$$
\begin{aligned}
A_{C}\left(\left\{x_{k}\right\}\right)= & \left\{x \in X: r\left(x,\left\{x_{k}\right\}\right) \leq r\left(y,\left\{x_{k}\right\}\right)\right. \\
& \text { for any } y \in C\} .
\end{aligned}
$$

If the asymptotic center is taken with respect to $X$. then it is simply denoted by $A\left(\left\{x_{k}\right\}\right)$.

A bounded sequence $\left\{x_{k}\right\}$ in $X$. is said to be regular if $r\left(\left\{x_{k}\right\}\right)=r\left(\left\{u_{k}\right\}\right)$ for every subsequence $\left\{u_{k}\right\}$ of $\left\{x_{k}\right\}$ Recall that a sequence $\left\{x_{k}\right\}$ converges weakly to $w$ (written as $x_{k} \rightarrow w$ ) if and only if

$r\left(w,\left\{x_{k}\right\}\right)=\inf _{x \in C} r\left(x,\left\{x_{k}\right\}\right)$, where $C$ is a closed and convex subset containing the bounded sequence $\left\{x_{k}\right\}$ Moreover, a sequence $\left\{x_{k}\right\}$ (in $X$.) $\Delta$-converges to $x \in X$ if $x$ is the unique asymptotic center of $\left\{u_{k}\right\}$ for every subsequence $\left\{u_{k}\right\}$ of $\left\{x_{k}\right\}$ In this case, we write $\Delta-\lim _{n} x_{n}=x$ and $x$ is called $\Delta$-limit of $\left\{x_{n}\right\}$.

In a Banach space setting, $\Delta$-convergence coincides with weak convergence. A connection between weak convergence and $\Delta$-convergence in geodesic spaces is characterized in the following lemma due to Nanjaras and Panyanak [26]. 
Lemma 2.4. ([26], Proposition 3.12). Let $\left\{x_{k}\right\}$ be a bounded sequence in a $C A T(0)$ space $X$. and let $C$ be a closed and convex subset of $X$. and contains $\left\{x_{k}\right\}$. Then

1) $\Delta-\lim _{k} x_{k}=x$ implies that $x_{k} \rightarrow x$,

2) the converse of (1) is true if $\left\{x_{k}\right\}$ is regular.

Next, we state the demiclosed principle in $\operatorname{CAT}(0)$ spaces due to Hussain and Khamsi [8] needed in the next convergence theorem.

Lemma 2.5. Let $C$ be a nonempty, bounded, closed and convex set in a CAT (0) space $X$. and $T: C \rightarrow C$ be a pointwise asymptotic nonexpansive map. Let $\left\{x_{k}\right\}$ be a sequence in $C$ such that $\left\{x_{k}\right\} \rightarrow \omega$ and $\lim _{k \rightarrow \infty} d\left(x_{k}, T x_{k}\right)=0$ Then $T(\omega)=\omega$.

Next, we prove our weak convergence theorem.

Theorem 2.6. Let $C$ be a nonempty, bounded, closed and convex set in a Hadamard space $X$. and let

$S, T \in \tau(C)$. Let $\alpha_{k}, \beta_{k} \in(\delta, 1-\delta)$ for some $\delta \in\left(0, \frac{1}{2}\right)$ and $\left\{n_{k}\right\}$ be such that the sequence $\left\{x_{k}\right\}$ in (1.2) is well defined. If the set $\Omega=\left\{j: n_{j+1}=n_{j}+1\right\}$ is quasiperiodic and $F \neq \varnothing$, then $\left\{x_{k}\right\}$ converges weakly to a point in $F$.

Proof. Let $\omega_{w}\left(x_{k}\right)$ be the weak $\omega$-limit set of $\left\{x_{k}\right\}$ given by

$$
\omega_{w}\left(x_{k}\right)=\left\{y \in C: x_{k_{i}} \rightarrow y \text { for }\left\{x_{k_{i}}\right\} \subseteq\left\{x_{k}\right\}\right\} .
$$

Since $C$ is a nonempty bounded closed convex subset of a Hadamard space, there exists a subsequence $\left\{x_{k_{i}}\right\}$ of $\left\{x_{k}\right\}$ such that $x_{k_{i}} \rightarrow p \in \omega_{w}\left(x_{k}\right)$ as $i \rightarrow \infty$ and vice versa. This shows that $\omega_{w}\left(x_{k}\right) \neq \varnothing$. As $x_{k_{i}} \rightarrow p$ and $\lim _{i \rightarrow \infty} d\left(x_{k_{i}}, S x_{k_{i}}\right)=0=\lim _{i \rightarrow \infty} d\left(x_{k_{i}}, T x_{k_{i}}\right)$ (by Lemma 2.1), therefore by Lemma 2.5, $S p=p=T p$. That is, $\omega_{w}\left(x_{k}\right) \subset F$. Next, we follow the idea of Chang et al. [14]. For any $p \in \omega_{w}\left(x_{k}\right)$, there exists a subsequence $\left\{x_{k_{i}}\right\}$ of $\left\{x_{k}\right\}$ such that

$$
x_{k_{i}} \rightarrow p \text { as } i \rightarrow \infty \text {. }
$$

Hence from (2.12) and (2.14), it follows that

$$
T^{n_{k_{i}}} X_{k_{i}} \rightarrow p \text { as } i \rightarrow \infty .
$$

Now from (1.2), (2.14) and (2.15), we get that

$$
y_{k_{i}}=\left(1-\beta_{k_{i}}\right) x_{k_{i}} \oplus \beta_{k_{i}} T^{n_{k_{i}}} x_{k_{i}} \rightarrow p \text { as } i \rightarrow \infty \text {. }
$$

Also from (2.12) and (2.14), we have that

$$
S^{n_{k_{i}}} y_{k_{i}} \rightarrow p \text { as } i \rightarrow \infty \text {. }
$$

Again from (1.2), (2.14) and (2.17), we conclude that

$$
x_{k_{i}+1}=\left(1-\alpha_{k_{i}}\right) x_{k_{i}} \oplus \alpha_{k_{i}} S^{n_{k_{i}}} y_{k_{i}} \rightarrow p .
$$

Continuing in this way, by induction, we can prove that, for any $l \geq 0$,

$$
x_{k_{i}+l} \rightarrow p
$$

By induction, one can prove that $\bigcup_{l=0}^{\infty}\left\{x_{k_{j}+l}\right\}$ converges weakly to $p$ as $j \rightarrow \infty$; in fact

$\left\{x_{k}\right\}_{k=k_{1}}^{\infty}=\bigcup_{l=0}^{\infty}\left\{x_{k_{j}+l}\right\}_{j=1}^{\infty}$ gives that $x_{k} \rightarrow p$ as $k \rightarrow \infty$.

Remark 2.7. If $\left\{x_{k}\right\}$ is regular in a geodesic space, then $\left\{x_{k}\right\}$ is $\Delta$-convergent.

Our strong convergence theorem is as follows. We do not use the rate of convergence condition namely

$\sum_{k=1}^{\infty}\left(c_{n_{k}}(x)-1\right)<\infty$ in its proof.

Theorem 2.8. Let $C$ be a nonempty, bounded, closed and convex set in a Hadamard space $X$ and let

$S, T \in \tau(C)$. Let $\alpha_{k}, \beta_{k} \in(\delta, 1-\delta)$ for some $\delta \in\left(0, \frac{1}{2}\right)$ and $\left\{n_{k}\right\}$ be such that the sequence $\left\{x_{k}\right\}$ in (1.2) is well defined. If the set $\Omega=\left\{j: n_{j+1}=n_{j}+1\right\}$ is quasiperiodic, $F \neq \varnothing$ and either $S$ or $T$ is semi-compact (completely continuous), then $\left\{x_{k}\right\}$ converges strongly to a point in $F$.

Proof. Let $S$ be semi-compact. As

$\lim _{k \rightarrow \infty} d\left(x_{k}, S x_{k}\right)=0$, there exists a subsequence $\left\{x_{k_{i}}\right\}$
of $\left\{x_{k}\right\}$ such that

$$
x_{k_{i}} \rightarrow p \text {. }
$$

Using $x_{k}=x_{k_{i}}$ in (2.13) and continuity of $S$ and $T$, we obtain that $p \in F$. The rest of the proof follows by replacing $\rightarrow$ with $\rightarrow$ in Theorem 2.6 and we, therefore, omit the details.

Finally, we state a theorem due to Nanjaras and Panyanak [26] proved in Hadamard spaces in which rate of convergence condition is necessary for $\Delta$-convergence of the sequence.

Theorem 2.9. Let $C$ be a nonempty, bounded, closed and convex set in a Hadamard space $X$ and let $T \in \tau(C)$ with a sequence $\left\{c_{k}\right\} \subset[1, \infty)$ for which $\sum_{k=1}^{\infty}\left(a_{k}-1\right)<\infty$. Suppose that $x_{1} \in C$ and $\left\{\alpha_{k}\right\}$ is a sequence in $(a, b)$ for some $a, b \in(0,1)$. Then the sequence $x_{k+1}=\left(1-\alpha_{k}\right) x_{k} \oplus \alpha_{k} T^{k} y_{k}, \Delta$-converges to a fixed point of $T$.

We pose the following open question.

Open question: Does Theorem 2.6 hold if we replace weak convergence by $\Delta$-convergence?

\section{REFERENCES}

[1] K. K. Tan and H. K. Xu, "Fixed Point Iteration Processes for Asymptotically Nonexpansive Mapping," Proceedings of the American Mathematical Society, Vol. 122, No. 3, 1994, pp. 733-739. doi:10.1090/S0002-9939-1994-1203993-5 
[2] M. Bridson and A. Haefliger, "Metric Spaces of Non-Positive Curvature," Springer-Verlag, Berlin, 1999.

[3] S. Dhompongsa and B. Panyanak, "On $\Delta$-Convergence Theorems in CAT(0) Spaces," Computers \& Mathematics with Applications, Vol. 56, No. 10, 2008, pp. 2572-2579. doi:10.1016/j.camwa.2008.05.036

[4] F. Bruhat and J. Tits, "Groupes Réductifs Sur Un Corps Local. I. Données Radicielles Valuées," Institut des Hautes Études Scientifiques Publications Mathématiques, Vol. 41, No. 1, 1972, pp. 5-251. doi:10.1007/BF02715544

[5] W. A. Kirk, "A Fixed Point Theorem in CAT(0) Spaces and R-Trees," Fixed Point Theory and Applications, Vol. 4, 2004, pp. 309-316.

[6] W. A. Kirk and H. K. Xu, "Asymptotic Pointwise Contractions," Nonlinear Analysis: Theory, Methods \& Applications, Vol. 69, No. 12, 2008, pp. 4706-4712. doi:10.1016/j.na.2007.11.023

[7] K. Goebel and W. A. Kirk, "A Fixed Point Theorem for Asymptotically Nonexpansive Mappings," Proceedings of the American Mathematical Society, Vol. 35, 1972, pp. 171-174. doi:10.1090/S0002-9939-1972-0298500-3

[8] N. Hussain and M. A. Khamsi, "On Asymptotic Pointwise Contractions in Metric Spaces," Nonlinear Analysis: Theory, Methods \& Applications, Vol. 71, No. 10, 2009, pp. 4423-4429. doi:10.1016/j.na.2009.02.126

[9] C. W. Groetsch, "A Note on Segmenting Mann Iterates," Journal of Mathematical Analysis and Applications, Vol. 40, No. 2, 1972, pp. 369-372. doi:10.1016/0022-247X(72)90056-X

[10] W. R. Mann, "Mean Value Methods in Iteration," Proceedings of the American Mathematical Society, Vol. 4, No. 3, 1953, pp. 506-510. doi:10.1090/S0002-9939-1953-0054846-3

[11] S. Ishikawa, "Fixed Points by a New Iteration Method," Proceedings of the American Mathematical Society, Vol. 44 , No. 1,1974 , pp. 147-150. doi:10.1090/S0002-9939-1974-0336469-5

[12] S. C. Bose, "Weak Convergence to the Fixed Point of an Asymptotically Nonexpansive," Proceedings of the American Mathematical Society, Vol. 68, No. 3, 1978, pp. 305-308. doi:10.1090/S0002-9939-1978-0493543-4

[13] R. Bruck, T. Kuczumow and S. Reich, "Convergence of Iterates of Asymptotically Nonexpansive Mappings in Banach Spaces with the Uniform Opial Property," Collectanea Mathematica, Vol. 65, No. 2, 1993, pp. 169-179.

[14] S. Chang, Y. J. Cho and H. Zhou, "Demiclosed Principle and Weak Convergence Problems for Asymptotically Nonexpansive Mappings," Journal of the Korean Mathematical Society, Vol. 38, 2001, pp. 145-1260.

[15] H. Fukhar-ud-din and S. H. Khan, "Convergence of TwoStep Iterative Scheme with Errors for Two Asymptotically Nonexpansive Mappings," International Journal of Mathematics and Mathematical Sciences, Vol. 2004, No. 37, 2004, pp. 1965-1971. doi:10.1155/S0161171204308161

[16] H. Fukhar-ud-din and S. H. Khan, "Convergence of Iterates with Errors of Asymptotically Quasi-Nonexpansive
Mappings and Applications," Journal of Mathematical Analysis and Applications, Vol. 328, No. 2, 2007, pp. 821-829. doi:10.1016/i.jmaa.2006.05.068

[17] H. Fukhar-ud-din and A. R. Khan, "Approximating Common Fixed Points of Asymptotically Nonexpansive Maps in Uniformly Convex Banach Spaces," Computers \& Mathematics with Applications, Vol. 53, No. 9, 2007, pp. 1349-1360. doi:10.1016/j.camwa.2007.01.008

[18] H. Fukhar-ud-din and A. R. Khan, "Convergence of Implicit Iterates with Errors for Mappings with Unbounded Domain in Banach Spaces," International Journal of Mathematics and Mathematical Sciences, Vol. 10, 2005, pp. 1643-1653. doi:10.1155/IJMMS.2005.1643

[19] H. Fukhar-ud-din, A. R. Khan, D. O'Regan and R. P. Agarwal, "An Implicit Iteration Scheme with Errors for a Finite Family of Uniformly Continuous Mappings," Functional Differential Equations, Vol. 14, No. 3-4, 2007, pp. 245-256.

[20] S. H. Khan and H. Fukhar-ud-din, "Weak and Strong Convergence of a Scheme with Errors for Two Nonexpansive Mappings," Nonlinear Analysis: Theory, Methods \& Applications, Vol. 61, No. 8, 2005, pp. 1295-1301. doi:10.1016/j.na.2005.01.081

[21] A. R. Khan and N. Hussain, "Iterative Approximation of Fixed Points of Nonexpansive Maps," Scientiae Mathematicae Japonicae, Vol. 54, No. 3, 2001, pp. 503-511.

[22] S. H. Khan and W. Takahashi, "Approximating Common Fixed Points of Two Asymptotically Nonexpansive Mappings," Scientiae Mathematicae Japonicae, Vol. 53, No. 1, 2001, pp. 143-148.

[23] W. M. Kozlowski, "Fixed Point Iteration Processes for Asymptotic Pointwise Nonexpansive Mappings in Banach Spaces," Journal of Mathematical Analysis and Applications, Vol. 377, No. 1, 2011, pp. 43-52. doi:10.1016/j.jmaa.2010.10.026

[24] T. Laokul and B. Panyanak, "Approximating Fixed Points of Nonexpansive Mappings in CAT(0) Spaces," International Journal of Mathematics and Mathematical Sciences, Vol. 3, No. 25-28, 2009, pp. 1305-1315.

[25] W. Laowang and B. Panyanak, "Approximating Fixed Points of Nonexpansive Nonself Mappings in CAT(0) Spaces," Fixed Point Theory and Applications, 2010, 11 pages, Article ID: 367274 .

[26] B. Nanjaras and B. Panyanak, "Demiclosed Principle for Asymptotically Nonexpansive Mapping in CAT(0) Spaces," Fixed Point Theory and Applications, 2010, 14 pages, Article ID: 268780.

[27] M. A. Noor and B. Xu, "Fixed Point Iterations for Asymptotically Nonexpansive Mappings in Banach Spaces," Journal of Mathematical Analysis and Applications, Vol. 267, No. 2, 2002, pp. 444-453. doi:10.1006/jmaa.2001.7649

[28] Z. Opial, "Weak Convergence of Successive Approximations for Nonexpansive Mappings," Bulletin of the American Mathematical Society, Vol. 73, 1967, pp. 591-597. doi:10.1090/S0002-9904-1967-11761-0

[29] G. B. Passty, "Construction of Fixed Points for Asymptotically Nonexpansive Mappings," Proceedings of the 
American Mathematical Society, Vol. 84, 1982, pp. 212216. doi:10.1090/S0002-9939-1982-0637171-7

[30] B. E. Rhoades, "Fixed Point Iterations for Certain Nonlinear Mappings," Journal of Mathematical Analysis and Applications, Vol. 183, No. 1, 1994, pp. 118-120. doi:10.1006/jmaa.1994.1135

[31] J. Schu, "Iterative Construction of Fixed Points of Asymptotically Nonexpansive Mappings," Journal of Ma- thematical Analysis and Applications, Vol. 158, No. 2, 1991, pp. 407-413.

doi:10.1016/0022-247X(91)90245-U

[32] J. Schu, "Weak and Strong Convergence to Fixed Points of Asymptotically Nonexpansive Mappings," Bulletin of the Australian Mathematical Society, Vol. 43, No. 1, 1991, pp. 153-159. doi:10.1017/S0004972700028884 